Ixora Requena 1 Yessica Hernández 1 Mario Ramsay 1 Carmen Salazar 1 Rodolfo Devera 1

\section{Prevalencia de Blastocystis hominis en vendedores ambulantes de comida del municipio Caroní, Estado Bolívar, Venezuela}

\author{
Prevalence of Blastocystis hominis among \\ food handlers from Caroni municipality, \\ Bolivar State, Venezuela
}

1 Departamento de Parasitología y Microbiología, Escuela de Medicina, Universidad de Oriente, Núcleo Bolívar. Av. José Méndez, Ciudad Bolívar, Estado Bolívar, 8001-A, Venezuela. rodolfodevera@hotmail.com
Abstract A cross-sectional survey was conducted to determine the prevalence of Blastocystis hominis infection in a random sample of apparently heal thy food handlers. A total of 415 individuals attending the Manoa Urban Outpatient Clinic (Caroní Municipality, Bolívar State, Venezuela) in the Adult Hygiene Program and who requested heal th certification to work as food handlers were studied. Stool samples obtained by spontaneous evacuation were examined by direct microscopy and the Willis concentration method. A total of 150 individuals were infected (36.14\%), 107 (25.78\%) of whom with B. hominis. There was no difference between males and females ( $p>0.05)$, but there was a significant difference between ages ( $\left.\chi^{2}=12.17 ; g . l .=4\right)$, with infection more frequent between 18 and 27 years. In $71.02 \%$ of the cases, B. hominis was the only parasite. Giardia lamblia was the parasi te most frequently associated with B. hominis (2.41\%). In the majority (85\%) of infected individuals, less than five microorganisms per microscopic field were observed. We conclude that B. hominis is a frequent intestinal parasite among food handlers in Caroní Municipality, Bolivar State, Venezuela.

Key words Blastocystis hominis; Prevalence; Food Handling

Resumen Para determinar la prevalencia de Blastocystis hominis en una muestra de vendedores ambulantes de comida, aparentemente sanos, se realizó un estudio seccional con 415 personas que acudieron al Ambulatorio Urbano tipo III "Manoa" (Municipio Caroní, Estado Bolívar, Venezuela), Programa de Higiene del Adulto, a solicitar el certificado de salud para trabajar como vendedores de comida. U na muestra de heces obtenida por evacuación espontán ea fue analizada mediante la técnica de examen directo y método de concentración de Willis. Se encontraron 150 personas parasitadas (36,14\%), de ellas 107 (25,78\%) con B. hominis. No se observó predilección por el sexo $(p>0,05)$, pero sí con relación a la edad, siendo las personas de 18 a 27 años las más afectados $\left(\chi^{2}=12,17 ;\right.$ g.l . =4). En el $71,02 \%$ de los casos se encontró como parási to único y en $28,98 \%$ de los casos asociados a otros parásitos, si endo el más frecuentemente asociado Giardia lamblia (2,41\%). En la mayoría de las personas parasitadas (85\%) el protozoario se observó en un número menor de cinco células por campo. Se concluye que B. hominis es un parásito frecuente en manipuladores de alimentos del Municipio Caroní, Estado Bolívar, Venezuela.

Palabras-clave Blastocystis hominis; Prevalencia; Manipulación de Alimentos 


\section{Introducción}

Los protozoarios constituyen un grupo numeroso e importante dentro de los parásitos intestinales, variando su prevalencia y patogenicidad de acuerdo a ciertos factores propios del agente o del hospedero (OMS, 1981). En los últimos años, con el advenimiento del Síndrome de Inmunodeficiencia Adquirida (SIDA), nuevos protozoarios han ocupado muchas páginas de la literatura mundial, son los Ilamados patógenos emergentes o reemergentes. Sin embargo, otros, siempre han estado presentes, pero es sólo ahora cuando se les reconoce como causantes de enfermedad humana. Blastocystis hominis es uno de ellos, se trata de un microorganismo muy complejo, confundido por mucho tiempo con artefactos o quistes de otros protozoarios, del cual aún persisten muchas controversias e incógnitas (Boreham \& Stenzel, 1993; Ponce-de-León et al., 1991; Zierdt et al., 1967). Se trata de un protozoario polimórfico del que se describen cuatro formas principales: forma de cuerpo central o vacuolada, granular, ameboide y el quiste. La primera es la más frecuentemente observada en heces constituyéndose por lo tanto en el estadio diagnóstico (Boreham \& Stenzel, 1993; Singh et al., 1995; Zaman et al., 1995; Zierdt et al., 1967). Diversos autores han mostrado que el examen directo de heces es un método práctico, barato y eficaz para el diagnóstico de los estadios evolutivos del parásito (Devera et al., 1998a; Pérez-de-Suárez \& Guzmán-de-Rondón, 1994; Pires-de-Mato et al., 1998), con excepción del quiste que, debido a su pequeño tamaño, requiere de técnicas especiales (Zaman, 1996).

Las pruebas experimentales de patogenicidad se apoyan en la inducción de diarrea en animales, la cual es similar a la que se produce en el hombre (Phillips \& Zierdt, 1976) y más recientemente se verificaron efectos patogénicos en ratones BALB/ c (Moe et al., 1998) y en cultivo de células (Walderich et al., 1998).

La enfermedad producida por el protozoario es conocida como Blastocistosis o enfermedad de Zierdt-Garavelli en honor a dos de los investigadores que más han estudiado al microorganismo (Boreham \& Stenzel, 1993).

Los síntomas más frecuentes incluyen diarrea, dolor abdominal, anorexia y flatulencia. También se describen otros no específicos de infección gastrointestinal. Generalmente, no se encuentran leucocitos fecales y se ha observado eosinofilia en algunos casos (Boreham \& Stenzel, 1993; Garavelli et al., 1989, 1991; García et al., 1984; Llibre et al., 1989; Sadek et al.,
1997; Sheehan et al., 1986; Vannatta et al., 1985; Wang et al., 2002).

Blastocitosis es una infección cosmopolita y el hombre no es el hospedero exclusivo, pues se han descrito Blastocystis morfológicamente similares a B. hominis en muchos vertebrados (Boreham \& Stenzel, 1993; Devera et al., 1999b), por lo que algunos autores la consideran una infección zoonótica, pero todavía se desconoce el rol de estos hospederos en la transmisión de protozoarios y si se trata o no de una especie única (Boreham \& Stenzel, 1993; Devera, 1998; Salin et al., 1999). Se acepta como mecanismo de infección la vía fecal-oral (Boreham \& Stenzel, 1993; Singh et al., 1995; Torres et al., 1992; Vannatta et al., 1985), similar a lo que ocurre con otros protozoarios intestinales. La ingestión de agua no tratada (Kain et al., 1987; M ejías, 1993; Torres et al., 1992) o de alimentos (Casemore, 1990; Garavelli \& Scaglione, 1989) contaminados con formas parasitarias ha sido sugerida.

Diversos estudios han mostrado cifras de prevalencia variables según el área geográfica y la población estudiada (Boreham \& Stenzel, 1993). En el estado Bolívar el estudio de grupos seleccionados de la población ha revelado tasas de prevalencias superiores a la media nacional que es en torno del $10 \%$ (Castrillo-de-Tirado et al., 1990; Devera et al., 1998b; Medrano \& Volcán, 1996).

El comercio informal es una realidad en todas las ciudades de Latinoamérica, siendo la venta de diversos tipos de alimentos una de las más difundidas. La falta de control higiénico de los alimentos vendidos por estas personas constituye un importante obstáculo a salvar cuando se quiere implementar medidas de control contra las parasitosis intestinales, pues son una de las fuentes principales de diseminación de enteropatógenos. En el Estado Bolívar, como en el resto de Venezuela, el poco control existente sobre los vendedores ambulantes de comida se limita a la realización del llamado Certificado de Salud, que no es más que una evaluación clínica general y exámenes de laboratorio (pesquisa del virus de la inmunodeficiencia humana (HIV), despistaje de Sífilis mediante serología y un examen coproparasitológico). Este certificado tiene una validez de un año y es realizado en centros reconocidos por el Ministerio de Salud y Desarrollo Social. Toda persona que manipule o venda alimentos en Venezuela debe poseer un certificado de salud vigente.

Considerando que la vía oro-fecal sea el principal mecanismo de transmisión de B. hominis y la alta frecuencia del protozoario en 
nuestra región, se diseñó un estudio para determinar la prevalencia de parasitosis intestinal, y en especial de $B$. hominis, en vendedores ambulantes de comida que solicitaron el Certificado de Salud en el Ambulatorio urbano tipo III “Manoa” en el Municipio Caroní, estado Bolívar, Venezuela, durante el periodo noviembre de 2000-abril de 2001.

\section{Materiales y método}

\section{Universo y muestra}

El universo estuvo representado por todas las personas adultas que solicitaron el Certificado de Salud en el Ambulatorio Urbano tipo III “Manoa", Programa de Higiene del Adulto, en el Municipio Caroní, durante el periodo noviembre de 2000-abril de 2001. La muestra estuvo formada por aquellas personas que declararon necesitar dicho certificado para ejercer el oficio de vendedores ambulantes de comidas.

\section{Recolección de datos}

\section{- Recolección y análisis de las heces}

De cada persona fue colectada una muestra fecal obtenida por evacuación espontánea. La muestra era dividida en dos porciones, una era analizada en el laboratorio de parasitología del ambulatorio mediante las técnicas de examen directo en solución salina $0,85 \%$ y lugol y el método de flotación en salmuera (Willis) (Melvin \& Brooke, 1971). La otra porción era preservada en frascos adecuados conteniendo Dicromato de potasio al $2 \%$. Estas muestras eran sometidas a las mismas técnicas en el Laboratorio de Diagnóstico Parasitológico de la Escuela de Medicina de la Universidad de Oriente.

- Morfología y cuantificación de B. hominis

En aquellas muestras donde se observó B. hominis, se determinó la morfología del protozoario y también se realizó su cuantificación. Para ello se contaron diez campos con objetivo de 40x, estando distribuidos sobre la preparación de la siguiente forma: dos en cada una de las esquinas, para un total de 8 , y dos en el centro de la lámina. Posteriormente se determinó un promedio de células del microorganismo observadas en esos campos, informándose el resultado como más de cinco células por campo o menos de cinco células por campo (Sheehan et al., 1986).

\section{- Evaluación clínica}

Además del examen coproparasitológico, cada persona fue sometida a una anamnesis orientada y un examen físico.

\section{Análisis de los datos}

Los resultados se analizaron mediante frecuencias relativas utilizando la distribución normal. La prevalencia se estimó según Morales \& Pino (1987). Se aplicó la prueba chi-cuadrado $\left(\chi^{2}\right)$ con corrección de Yates, con un intervalo de confianza del 95\% para determinar la relación entre parasitosis y edad (M orales \& Pino, 1987).

\section{Consideraciones éticas}

Este trabajo fue aprobado por la Comisión de Tesis de la Escuela de Ciencias de la Salud, Universidad de Oriente, que evalúa factibilidad, pertinencia, rigor científico y aspectos éticos de cada proyecto sometido a consideración. Todos los participantes del estudio dieron su consentimiento por escrito. Además, los individuos que resultaron parasitados fueron tratados con drogas antiparasitarias específicas.

\section{Resultados}

Durante el período estudiado un total de 415 muestras de heces de igual número de personas fueron evaluadas, la edad osciló entre 18 y 72 años, con 260 del sexo femenino y 155 del sexo masculino. La prevalencia general de parasitosis intestinales fue de $36,14 \%$ al encontrarse 150 infectados por alguna especie de protozoario o helminto intestinal. En la Tabla 1 se muestran las prevalencias de los diferentes parásitos y/ o comensales intestinales encontrados. Los protozoarios resultaron más frecuentes que los helmintos, siendo $\mathrm{B}$. hominis el más prevalente con 25,78\% (107/415), seguido de Giardia Iamblia y Entamoeba coli con $2,40 \%$ cada uno. Entre los helmintos, apenas se diagnosticaron cinco casos (1,20\%) de A. Iumbricoides y cuatro $(0,96 \%)$ de T. trichiura, resultando éstos los helmintos más frecuentes.

La infección por $B$. hominis fue más común en el grupo de edad de 18 a 27 , con $48,60 \%$ de los casos, siendo esta diferencia estadísticamente significativa $\left(\chi^{2}=12,17 ;\right.$ g.l. $\left.=4\right)$. No hubo predilección por el sexo ( $p>0,05)$, resultando parasitados el $57,94 \%$ de las mujeres y $42,06 \%$ de los hombres (Tabla 2 ).

De los 107 casos de infección por B. hominis, en $91(85,00 \%)$ se observaron menos de 
cinco células del protozoario por cada diez campos de $400 X$ y apenas 16 (15,00\%) con un número mayor a cinco células por campo. La única forma identificada fue la vacuolar.

El protozoario se identificó principalmente como parásito único $(71,00 \%)$ y en los 31 casos $(29,00 \%)$ donde estuvo asociado a otras especies parasitarias y/o comensales la asociación más frecuente fue con G. Iamblia (Tabla 3).

El $42,00 \%$ de las personas estaba asintomático y $58,00 \%$ sintomático.

\section{Discusión}

Aunque considerado durante mucho tiempo un comensal inocuo del intestino, B. hominis es actualmente un parásito intestinal de preva-

Tabla 1

Prevalencia de parásitos intestinales en vendedores ambulantes de comida del Municipio Caroní, Estado Bolívar, Venezuela. Noviembre 2000-Abril 2001.

\begin{tabular}{lrr}
\hline Parásito* & $\mathrm{n}$ & $\%$ \\
\hline Blastocystis hominis & 107 & 25,78 \\
Giardia lamblia & 10 & 2,41 \\
Entamoeba coli & 10 & 2,41 \\
Chilomastix mesnilii & 6 & 1,44 \\
Ascaris lumbricoides & 5 & 1,20 \\
Trichuris trichiura & 4 & 0,96 \\
Entamoeba histolytica/E. dispar & 4 & 0,96 \\
Anquilostomideos & 3 & 0,72 \\
Strongyloides stercoralis & 1 & 0,24 \\
\hline
\end{tabular}

* Se incluyen protozoarios comensales. lencia creciente del cual aun se desconocen muchos aspectos epidemiológicos (Boreham \& Stenzel., 1993; Ponce-de-León et al., 1991; Sheehan et al., 1986). Algunos autores han sugerido que el mecanismo de transmisión es la vía fecal-oral (Boreham \& Stenzel, 1993; Senay \& MacPhearson, 1990; Singh et al., 1995; Torres et al., 1992); además, los estudios de prevalencia han mostrado resultados muy variables (Boreham \& Stenzel, 1993). En el presente estudio se determinó la prevalencia de parasitosis intestinal y particularmente la de $B$. hominis en manipuladores de alimentos. Un total de 415 personas adultas que acudieron a solicitar el Certificado de Salud para desempeñarse como vendedores ambulantes de comida fueron evaluados coproparasitológicamente. Todos asistieron a la Consulta de Higiene del Adulto del Ambulatorio Urbano Tipo III "Manoa" en el Municipio Caroní, en el estado Bolívar, Venezuela. Se determinó un relativo alto índice de parasitosis intestinales $(36,14 \%-150 / 415)$ así como una mayor prevalencia de protozoarios $(33,01 \%$ - 137/415). Estos resultados son alarmantes si se considera que se trata de un grupo de alto riesgo de transmisión debido a las actividades de manipulación de alimentos que realizan. Hallazgos similares han sido señalados en otros estudios (Amin, 1997; Benetton et al., 1999).

Se estimó una prevalencia de $\mathrm{B}$. hominis de $25,78 \%$ la cual es mayor a la determinada en otros grupos de la población tanto en el estado Bolívar (Devera et al., 1998a, 1998b; M edrano \& Volcán, 1996) como en otras localidades de Venezuela (Castrillo-de-Tirado et al., 1990; De Abreu et al., 1990; Pérez et al., 1996).

Tabla 2

Vendedores ambulantes de comida con Blastocystis hominis, según edad y sexo.

Municipio Caroní, Estado Bolívar, Venezuela. Noviembre 2000-Abril 2001.

\begin{tabular}{|c|c|c|c|c|c|c|}
\hline \multirow{3}{*}{$\begin{array}{l}\text { Grupos de edad } \\
\text { (años) }\end{array}$} & \multicolumn{4}{|c|}{ Sexo } & \multicolumn{2}{|c|}{ Total } \\
\hline & \multicolumn{2}{|c|}{ Femenino } & \multicolumn{2}{|c|}{ Masculino } & \multirow[b]{2}{*}{$\mathrm{n}$} & \multirow[b]{2}{*}{$\%$} \\
\hline & $n$ & $\%$ & $n$ & $\%$ & & \\
\hline $18-27$ & 24 & 22,43 & 28 & 26,17 & 52 & 48,60 \\
\hline $28-37$ & 15 & 14,02 & 8 & 7,48 & 23 & 21,50 \\
\hline $38-47$ & 13 & 12,15 & 5 & 4,67 & 18 & 16,82 \\
\hline $48-57$ & 7 & 6,54 & 4 & 3,74 & 11 & 10,28 \\
\hline$\geq 58$ & 3 & 2,80 & 0 & 0,00 & 3 & 2,80 \\
\hline Total & 62 & 57,94 & 45 & 42,06 & 107 & 100,00 \\
\hline
\end{tabular}

$\chi^{2}=12,17 ; g . I .=4 ; p<0,05$. 
El grupo de adultos jóvenes (18-27 años) resultó el más afectado, declinando la prevalencia conforme aumentó la edad de los evaluados. Algunos autores sostienen que los adultos son los más afectados (Ashford \& Atkinson, 1992; Boreham \& Stenzel, 1993; Doyle et al., 1990) aunque otros estudios han mostrado que los niños también presentan elevadas tasas de prevalencia (Devera et al., 1999a; Ponce-deLeón et al., 1991). Esto no pudo ser verificado en el presente estudio pues todos los evaluados eran adultos. Con relación al sexo, no hubo diferencia estadísticamente significativa entre hombres y mujeres parasitadas por el protozoario, coincidiendo con otros autores (De Abreu et al., 1990; Devera et al., 1998a, 1998b; Ponce-de-León et al., 1991; Torres et al., 1992; Wang et al., 2002).

Cabe resaltar la asociación de $\mathrm{B}$. hominis con G. Iamblia con la cual pudiera compartir igual mecanismo de transmisión y algunos sugieren hasta un sinergismo y mecanismos patogénicos similares (Ponce-de-León et al., 1991). Esta asociación ha sido señalada previamente (Castrillo-de-Tirado et al., 1990; Devera et al., 1998b; Ponce-de-León et al., 1991; Torres et al., 1992).

Aunque más de la mitad de las personas refirieron síntomas, se debe recalcar que ellas acudieron a una consulta de personas aparentemente sanas a solicitar un certificado de salud; además, la mayoría de los síntomas fueron de índole general e inespecífica que no necesariamente obedece a la infección por B. hominis. Por otra parte, el $85,00 \%$ de los parasitados presentaron menos de cinco células del protozoario por cada diez campos microscópicos de $400 x$ observados, lo cual puede explicar la escasez de manifestaciones clínicas y hasta la inespecificidad de los síntomas. Se ha informado que las personas con cargas parasitarias elevadas son las que presentan mayor sintomatología (De Carli \& Rott, 1994; Devera et al., 1998a; Doyle et al., 1990; García et al., 1984; Kain et al., 1987; Ponce-de-León et al., 1991; Sheehan et al., 1986; Vannatta et al., 1985)

En Venezuela no se conoce cuáles son las cifras de prevalencia de $B$. hominis entre manipuladores de alimento, siendo pocos los estudios realizados a ese respecto. Díaz et al. (1996) en Maracaibo, al occidente del país, evaluaron cien expendedores de alimentos que laboraban en las cantinas escolares de los colegios públicos y privados de esa ciudad obteniendo una prevalencia de 53,00\%, mayor a la determinada en el presente estudio. Estos autores determinaron una prevalencia de parasitosis muy elevada $(76,00 \%)$ y como en nuestro trabajo, los
Tabla 3

Frecuencia de asociaciones parasitarias con Blastocystis hominis en vendedores ambulantes de comida, Municipio Caroní, Estado Bolívar, Venezuela. Noviembre 2000-Abril 2001.

\begin{tabular}{lcr}
\hline B. hominis con & $\mathrm{n}$ & $\%$ \\
\hline Giardia lamblia & 8 & 25,81 \\
Chilomastix mesnilii & 6 & 19,36 \\
Entamoeba coli & 5 & 16,12 \\
Ascaris lumbricoides & 5 & 16,12 \\
Anquilostomideos & 3 & 9,68 \\
Trichuris trichiura & 3 & 9,68 \\
Entamoeba histolytica/E. dispar & 1 & 3,23 \\
Total & 31 & 100,00 \\
\hline
\end{tabular}

protozoarios predominaron en la muestra estudiada. Se sabe que la transmisión de los protozoarios se realiza principalmente por vía hídrica y alimentos contaminados, resultando más afectados los adultos (OMS, 1981).

En otros países el problema de los manipuladores de alimentos como potenciales diseminadores de enteroparásitos también ha sido poco estudiado. En Brasil, se evaluaron 264 individuos que manipulaban alimentos en 57 escuelas de la ciudad de Uberlândia, destacando la baja prevalencia de parasitosis y la ausencia de B. hominis (de Rezende et al., 1997). Estos autores emplearon la técnica de sedimentación espontánea que se sabe utiliza agua corriente, la cual lisa las formas vacuolares de $B$. hominis (De Carli \& Rott, 1994; Devera et al., 1999a; Melvin \& Brooke, 1971).

También en Brasil, Benetton et al. (1999) encontraron una elevada prevalencia de $80 \%$ para B. hominis entre manipuladores de alimentos en ferias libres, cifras éstas bien mayores a la determinada por nosotros. En Egipto, Sadek et al. (1997) realizaron un estudio dirigido específicamente a determinar la prevalencia de B. hominis en 1.700 manipuladores de alimentos del sexo masculino, verificando que el $8 \%$ estaba parasitado por el protozoario, lo cual representa una prevalencia tres veces menor a la señalada en el presente estudio. En Arabia Saudita, Amin (1997) determinó una prevalencia de parasitosis intestinal de $36,00 \%$ en 250 manipuladores de alimentos mayores de 21 años, que también es una cifra menor a la determinada por nosotros, sin embargo, la prevalencia de $B$. hominis en ese grupo fue mayor $(22,20 \%)$.

En conclusión, se determinó una elevada prevalencia de infección por B. hominis entre 
vendedores de comidas ambulantes del municipio Caroní en el estado Bolívar, Venezuela. Por lo tanto, este debe ser un grupo prioritario a ser considerado cuando se realicen medidas de control de estas parasitosis en la población. Recomendamos realizar un seguimiento y control de cura parasitológica, después del trata-

\section{Agradecimientos}

A la Lic. Mireya León y a todo el personal del laboratorio del Ambulatorio Urbano Tipo III "Manoa" por su colaboración.

\section{Referencias}

AMIN, A. M., 1997. Blastocystis hominis among apparently healthy food handles in Jeddah, Saudi Arabia. Journal of the Egyptian of Society of Parasitology, 27:817-823.

ASHFORD, R. W. \& ATKINSON, E. A., 1992. Epidemiology of Blastocystis hominis infection in Papua New Guinea: Age prevalence and association with others parasites. Annals of Tropical and Medical Parasitology, 86:29-136.

BENETTON, M. L.; PINHEIRO, S.; MACHADO, P.; PAES, M.; SILVA, R. \& ODA, W., 1999. Prevalencia parasitaria em manipuladores de alimentos em feiras livres da cidade de Manaus. Revista da Socidade Brasileira de Medicina Tropical, 32(Sup. 1):308-309.

BOREHAM, L. P. F. \& STENZEL, D. J., 1993. Blastocystis in humans and animals: Morphology, biology, and epizootology. Advances in Parasitology, 32:170.

CASEM ORE, D. P., 1990. Foodborne protozoal infection. Lancet, 336:1427-1432.

CASTRILLO-DE-TIRADO, A.; GONZÁLEZ, M. A. J. \& TIRADO, E., 1990. Frecuencia de infección por Blastocystis hominis: Un año de estudio. GEN (Revista de la Sociedad Venezolana de Gastroenterología), 44:217-220.

DE ABREU, A.; GALINDO, G.; MARTÍNEZ, D.; NÚNEEZ, C.; BASTIDAS, E.; PACHECO, M. \& AGUILAR, C. M., 1990. Infección por Blastocystis hominis en el Caserío Las Rosas Comunidad Rural del Estado Cojedes. Acta Científica Venezolana, 41(Sup. 1):276.

DE CARLI, G. A. \& ROTT, M. B., 1994. Blastocystis hominis e o exame parasitológico das fezes. Revista Brasileira de Análises Clinicas, 26:89-90.

DE REZENDE, C. H.; COSTA-CRUZ, J. M. \& GEN NARI-CARDOSO, M. L., 1997. Enteroparasitoses em manipuladores de alimentos das escolas públicas de U berlândia (Minas Gerais), Brasil. Revista Panamericana de Salud Pública, 2:392-397. miento específico, y no expedir el Certificado de Salud hasta que se demuestre la ausencia de formas parasitarias en las heces de estas personas. Finalmente, otros estudios son necesarios para evaluar los factores epidemiológicos determinantes de la elevada prevalencia de blastocistosis en este grupo de personas.
DEVERA, R., 1998. Blastocystis hominis: O enigma continua. Revista da Socidade Brasileira de Medicina Tropical, 31:491-492.

DEVERA, R.; CERRÓN, I.; GONZÁLEZ, J.; REQUENA, I.; CASTILLO, H. \& VELÁSQUEZ, V., 1999a. Blastocystis hominis en el laboratorio de Parasitología de la Escuela de Medicina de la Universidad de Oriente, periodo 1986-1995. Ciudad Bolívar, Venezuela. In: XV Jornadas Científicas, Tecnológicas y Educativas de Guayana, Memorias, pp. 70-71, Ciudad Bolívar: Asociación para el Avance de la Ciencia.

DEVERA, R.; NIEBLA-PUNOS, G.; NASTASI, J. A.; VELÁSQUEZ, V. J. \& GONZÁLEZ-MENESES, R., 1998a. Prevalencia de infección por Blastocystis hominis en niños del Estado Bolívar: Valor del examen directo de heces en el diagnóstico. In: XV Jornadas Científicas, Tecnológicas y Educativas de Guayana, Memorias, pp. 28-29, Ciudad Bolívar: Asociación para el Avance de la Ciencia.

DEVERA, R.; REQUENA, I.; VELÁSQUEZ, V.; CASTILLO, H. \& GONZÁLEZ, R., 1999b. Cerdos como reservorios de Blastocystis spp. en una comunidad rural del Estado Bolívar, Venezuela. Enfermedades Infecciosas y Microbiología Clínica, 17:422.

DEVERA, R. A.; VELÁSQUEZ, V. J . \& VÁSQUEZ, M. J., 1998b. Blastocistosis en pre-escolares de Ciudad Bolívar, Venezuela. Cadernos de Saúde Pública, 14: 401-407.

DíAZ, A. I.; ÁlVAREZ. J.; BETANCOURT, L.; LEAM OUTH, F. \& VILCHEZ, M. J., 1996. Enteroparasitosis en muestras fecales y sub-ungueales de manipuladores de alimentos. In: XIII Congreso Latinoamericano de Microbiología; VI Congreso Venezolano de Microbiología, Resúmenes, p. 137, Caracas: Asociación Latinoamericana de Microbiología/ Sociedad Venezolana de Microbiología.

DOYLE, P. W.; HELHGASON, M. M.; MATRIAS, R. G. \& PROCTOR. E. M., 1990. Epidemiology and 
pathogenecity of Blastocystis hominis. Journal of Clinical Microbiology, 28:116-121.

GARAVELLI, P. L. \& SCAGLIONE, L., 1989. Blastocystis and epidemiological study. Microbiológica, 12: 158.

GARAVELLI, P. L.; SCAGLIONE, L.; BICOCCHI, R. \& LIBANORE, M., 1991. The pathogenecity of Blastocystis hominis. Lancet, 5:76-79.

GARAVELLI, P. L.; SCAGLIONE, L.; ROSSI, M. R.; BICOCCHI, R. \& LIBANORE, M., 1989. Blastocystis in Italy. Annales de Parasi tologie Humaine et Comparee, 64:391-395.

GARCÍA, L. S.; BRUCKNER, D. A. \& CLANCY, M. N., 1984. Clinical relevance of Blastocystis hominis. Lancet, i:1233-1234.

KAIN, K. C.; NOBLE, M. A.; FREEMAN, H. J. \& BARTELUK, R. L., 1987. Epidemiology and clinical features associated with Blastocystis hominis infection. Diagnostic Microbiology and Infectious Disease, 8:235-244.

LLIBRE, J. M.; TOR, J.; MONTEROLA, J. M.; CARBONELL, C. \& FOZ, M., 1989. Blastocystis hominis chronic diarrhoea in AIDS patients. Lancet, i:221.

MEDRANO, C. E. \& VOLCAN, G. S., 1996. Coproparasitología en escolares indígenas y mestizos del Estado Bolívar, Venezuela. In: XIII Congreso Latinoamericano de Microbiología, VI Congreso Venezolano de Microbiología, Resúmenes, p. 103, Caracas: Asociación Latinoamericana de Microbiología/Sociedad Venezolana de Microbiología.

MEJÍAS, G. 1993. Infecciones enteroparasitarias en escolares rurales del Archipiélago de Chiloe, $X$ Región, Chile. Boletín Chileno de Parasitología, 48:28-29.

MELVIN, D. M.\& BROOKE, M. M., 1971. Métodos de Laboratorio para Diagnóstico de Parasitosis Intestinales. M éxico, DF: Nueva Editorial Interamericana.

MOE, K. T.; SINGH, M.; GOPA, A.; AKRISHNAKONE, L. C.; HO, L. C.; TAN, S. W.; CHEN, X. Q. \& YAP, E. H., 1998. Cytopathic effect of Blastocystis hominis after intramuscular inoculation into laboratory mice. Parasitology Research, 84:450-454.

MORALES, G. \& PINO, L. A., 1987. Parasitología Cuantitativa. Caracas: Fondo Editorial Acta Científica Venezolana.

OMS (Organización Mundial de la Salud), 1981. Infecciones Intestinal es por Protozoos y Hel mintos. Serie Informes Técnicos 666. Geneva: OMS.

PÉREZ, B. C. J.; RICHANY, J. L. M.; SEARA, T. E. J. \& SERJEAUNT, C. S. M., 1996. Blastocystis hominis: Su clínica relacionada con la morfología y la cantidad de parásitos en las heces. Acta Científica Venezolana, 47(Sup. 1):225.

PÉREZ-DE-SUÁREZ, E. \& GUZMÁN-DE-RONDÓN, C., 1994. La morfología del Blastocystis hominis en las heces y evaluación de métodos parasitológicos. GEN (Revista dela Sociedad Venezolana de Gastroenterología), 48:226-231.

PHILLIPS, B. P. \& ZIERDT, C. H., 1976. Blastocystis hominis: Pathogenic potencial in human patients and in gnotobiotes. Experimental Parasitology, 39:358-364.

PIRES-DE-MATO, C.; AM ATO NETO, V.; BRAZ, L. M. A.; CARIGNANI, F. L.; VILLELA, M. S. H.; PINTO, T. H. L.; DI PIETRO-FERNANDES, A. O. \& DE
MARCHI-CASADEI, R. M., 1998. Bastocystis hominis. Diagnóstico por exame direto e por coloração com tionina. Revista da Soci edade Brasileira de Medicina Tropical, 31(Sup. 1):188.

PONCE-DE-LEÓN, P.; SVETAZ, M. J. \& ZEDERO, M., 1991. Importancia del diagnóstico de Blastocystis hominis en el examen parasitológico de heces. Revista Latino-Americana de Microbiología, 33: 156-164.

SADEK, Y.; el-FAKAHANY, A. F.; LASHIN, A. H. \& elSALAM, F. A., 1997. Intestinal parasites among food-handlers in Qualybia Governorate, with reference to the pathogenic parasite Blastocystis hominis. Journal of the Egyptian of Society of Parasitology, 27:471-478.

SALIN, R. H.; KUMAR, G. S.; VELLAYAN, S.; MAK, J. W.; ANUAR, A. K.; INIT, I.; VENNILA, G. D.; SAMINATHAN, R. \& RAM AKRISHMAN, K., 1999. Blastocystis in animal handlers. Parasitology Reasearch, 85:1032-1033.

SENAY, H. \& MacPHERSON, D., 1990. Blastocystis hominis: Epidemiology and natural history. Journal of Infectious Diseases, 162:987-990.

SHEEHAN, D. J.; RAUCHER, B. G. \& MCKITRICK, J. C., 1986. Association of Blastocystis hominis with signs and symptoms of human disease. Journal of Clinical Microbiology, 24:548-550.

SINGH, M.; SURESH, K.; HO, L. C.; NG, G. C. \& YAP, E. $H ., 1995$. Elucidation of the life cycle of the intestinal protozoan Blastocystis hominis. Parasitol ogy Research, 81:446-450.

TORRES, P.; MIRANDA, J.; FLORES, L.; RIQUELME, J.; FRANJOLA, R. \& PÉREZ, J., 1992. Blastocystis y otras infecciones por protozoarios intestinales en comunidades humanas ribereñas de la cuenca del río Valdivia, Chile. Revista do Instituto de Medicina Tropical de São Paulo, 34:557-564.

VANNATTA, J.; ADAM SON, D. \& MULLIGAN, K., 1985. Blastocystis hominis infection presenting as recurrente diarrea. Annals of Internal Medicine, 102:495-496.

WALDERICH， B.; BERNAUER， S.; RENNER, M.; KNOBLOCH, J. \& BUCHARD, G. D., 1998. Cytopathic effects of Blastocystis hominis on Chinese hamster ovary $(\mathrm{CHO})$ and adenocarcinoma HT29 cell cultures. Tropical Medicine and International Health, 3:385-390.

WANG, K. X.; LI, C. P.; WANG, J. \& CUI, Y. B., 2002. Epidemiological survey of Blastocystis hominis in Huainan City, Anhui Province, Chine. World Journal of Gastroenterolgy, 8:928-932.

ZAM AN, V., 1996. The diagnosis of Blastocystis hominis. Journal of Infection, 33:15-16.

ZAM AN, V.; HOWE, J. \& NG, M., 1995. Ultrastructure of Blastocystis hominis cysts. Parasitology Research, 81:465-469.

ZIERDT, C. H.; RUDE, W. S. \& BULL, B. S., 1967. Protozoan characteristics of Blastocystis hominis. American Journal of Clinical Pathology, 48:495501.

Recibido el 25 de noviembre de 2002

Versión final presentada el 18 de junio de 2003

Aprobado el 31 de julio de 2003 